Service social

\title{
Une nouvelle mesure pour analyser la pauvreté au Québec : l'indice synthétique de pauvreté-richesse
}

\section{François Gardes et Simon Langlois}

Volume 44, numéro 3, 1995

Pauvreté

URI : https://id.erudit.org/iderudit/706705ar

DOI : https://doi.org/10.7202/706705ar

Aller au sommaire du numéro

\section{Éditeur(s)}

École de service social de l'Université Laval

ISSN

1708-1734 (numérique)

Découvrir la revue

Citer cet article

Gardes, F. \& Langlois, S. (1995). Une nouvelle mesure pour analyser la pauvreté au Québec : l'indice synthétique de pauvreté-richesse. Service social, 44(3), 29-53. https://doi.org/10.7202/706705ar
Résumé de l'article

Cet article propose une nouvelle mesure de la pauvreté: l'indice synthétique de pauvreté-richesse (ISPR). Cet indice est multidimensionnel, les trois dimensions retenues étant le revenu, les dépenses totales et la privation des ménages. Cet indice est à la fois absolu et relatif. Le caractère relatif de la pauvreté a été opérationnalisé en fonction de la région, de l'âge et de la scolarité. Les propriétés formelles de cet indice rendent possibles les comparaisons internationales et les analyses temporelles sur l'évolution du phénomène de la pauvreté, sans biais systématique. Le but de cette mesure ne vise pas avant tout un comptage du nombre des pauvres, mais bien l'élaboration d'un instrument d'analyse de la pauvreté. Les données canadiennes analysées proviennent de l'Enquête sur les dépenses des familles de Statistique Canada. La pauvreté reste élevée au Québec, mais l'écart avec l'Ontario s'amenuise fortement, puisque la pauvreté y est presque aussi élevée qu'au Québec. La pauvreté est aussi en progression dans le groupe des ménages les plus jeunes et les moins scolarisés. 


\section{Une nouvelle mesure pour analyser la pauvreté au Québec: I'indice synthétique de pauvreté-richesse ${ }^{7}$}

François GARDES

Professeur, Université de Paris-Sorbonne et Laboratoire de microéconomie appliquée (Lamia)

Simon LANGLOIS Professeur, Département de sociologie, Université Laval

La mesure du phénomène de la pauvreté pose de nombreux problèmes théoriques, méthodologiques et même politiques, au point de faire l'objet de débats dans les journaux et sur la place publique. Plusieurs raisons expliquent l'insatisfaction ressentie devant les mesures existantes. Tout d'abord, la pauvreté elle-même a changé. Les recherches qualitatives menées sur le terrain ont mis en évidence de nouvelles formes de pauvreté (Paugam, 1994, 1996; Nolan et Callan, 1994) et de nouveaux visages de la pauvreté (Gauthier, 1987 ; Conseil économique du Canada, 1992), mais les mesures quantitatives de ce phénomène à l'échelle de toute la société ont progressé plus 
timidement (Citro et Michael, 1995). Par ailleurs, les mesures existantes ne parviennent pas à prendre en considération les mutations culturelles, sociales et économiques du monde contemporain et elles paraissent incapables de tenir compte des nouvelles dimensions que la pauvreté a prises depuis plus de vingt ou trente ans. Nous proposons de combler cette lacune en construisant un nouvel indice de pauvreté - un indice qui mesurera aussi en même temps la richesse -, indice construit surtout dans le but de mener des analyses du phénomène de la pauvreté définie selon une approche multidimensionnelle, alors que les mesures principales sont en majorité, sinon en totalité, unidimensionnelles.

Nous passerons d'abord en revue les mesures existantes, afin de faire ressortir leurs limites et leurs faiblesses, avant d'expliciter les paramètres de la construction de l'indice de pauvreté-richesse (ISPR) que nous proposons. Cet article aura surtout un caractère méthodologique, certes, mais nous essaierons de réduire au strict minimum les détails techniques, afin d'accorder une place à la présentation d'un certain nombre de résultats empiriques obtenus.

\section{Mesures de la pauvreté des ménages}

Les chercheurs ne s'entendent pas sur la meilleure façon de mesurer le phénomène de la pauvreté (Goedhart, Halberstadt et van Praag, 1977 ; Greer et Thorbeck 1984 ; Ringen, 1987 ; Hagenaars, 1987 ; Hagenaars et de Vos, 1988; Foster et Shorrocks, 1991; Callan, Nolan et Whelan, 1993; Alcock, 1993). Ces désaccords viennent du fait qu'il existe des définitions différentes de ce phénomène social, mais aussi de ce que les mesures proposées poursuivent des objectifs qui ne sont pas les mêmes (Marklund, 1990; Lesemann, 1995). Les mesures de la pauvreté peuvent être classées en deux grandes catégories: celles qui visent à estimer la proportion d'une population qui tombe sous un seuil donné de pauvreté et celles qui caractérisent la gravité de la pauvreté et la distance qui sépare les pauvres entre eux, de même que les pauvres et les non-pauvres.

Les seuils de pauvreté sont définis selon différentes approches. Trois critères sont principalement utilisés pour construire ces mesures qui cherchent à estimer des proportions de pauvres. Le revenu disponible par unité de consommation est un critère fréquemment retenu: seront pauvres les ménages qui ont moins que la moitié de la moyenne nationale ou encore moins que la moitié de la médiane. Le seuil de pauvreté Eurostat est construit de cette façon. D'autres mesures de la pauvreté ont été élaborées à partir d'un ensemble 
$d^{\prime}$ indicateurs de conditions de vie ou de privations: absence d'une salle de bain ou de l'équipement de base du logement, conditions de salubrité, environnement, nombre de pièces par personnes, etc., indicateurs qui sont regroupés dans un indice additif. Seront pauvres les ménages qui se situent aux échelons inférieurs sur cet indice. L'OCDE a mis au point un indicateur de privation matérielle de ce type. Enfin, on a aussi estimé des seuils subjectifs de pauvreté à partir de questions d'enquêtes diversement formulées sur le revenu minimum jugé nécessaire par le ménage pour vivre à l'aise ou pour arriver dans son budget quotidien. Ces trois critères sont cependant le plus souvent considérés isolément, ce qui rend la majorité des mesures proposées incapables de rendre compte du caractère pluridimensionnel du phénomène de la pauvreté. Au Canada, tout comme au Québec, les deux mesures les plus connues et les plus utilisées par les chercheurs sont le Seuil de faible revenu (SFR) et la Mesure de faible revenu (MFR) de Statistique Canada.

Le SFR est déterminé à partir des dépenses moyennes des ménages observées pour trois postes fondamentaux: I'alimentation, le logement et l'habillement. L'organisme statistique considère comme étant à faible revenu les ménages qui consacrent à ces trois fonctions une part de leur budget supérieure de $20 \%$ à la moyenne nationale. En 1969, étaient considérés comme étant à faible revenu les ménages qui consacraient plus de $62 \%$ de leurs dépenses totales à ces trois fonctions de consommation. Les proportions ont diminué à $58,5 \%$ en 1978 , à $56,2 \%$ en 1986 et à $54,7 \%$ en 1992. Le SFR est un seuil mixte, à la fois absolu et relatif. Absolu parce qu'il est estimé à partir des dépenses les plus essentielles des ménages et relatif parce qu'il tient compte de l'enrichissement des ménages comme l'indique la décroissance de la proportion.

Plusieurs critiques ont été faites de ce seuil SFR (Langlois, 1987; Fugère et Lanctôt, 1985; Wolfson et Evans, 1990). Nous nous contenterons ici d'en faire état. Le revenu de référence comprend les paiements de transferts, mais il ne tient pas compte de l'effet redistributeur de l'impôt sur le revenu personnel. Statistique Canada considère l'impôt direct comme une dépense, alors qu'il s'agit d'une ponction qui vient réduire le revenu et la consommation des individus. Le calcul du cœfficient d'effort s'en trouve faussé. Ce problème est particulièrement important pour l'étude de l'évolution temporelle du phénomène de la pauvreté, parce que le taux moyen d'imposition est passé au Québec de $9 \%$ à environ $30 \%$ entre 1960 et 1996. Pour cette raison, le revenu disponible doit être préféré au revenu brut. Le seuil SFR tient compte de l'enrichissement moyen de la 
population, mais on peut critiquer la façon dont il mesure le caractère absolu de la faiblesse du revenu, notamment parce que le panier de base de dépenses qui est considéré est incomplet. Les besoins changent dans le temps et le panier de base doit inclure à l'aube de I'an 2000 des dépenses qui n'étaient pas jugées nécessaires dans les années 1960, comme les dépenses pour le téléphone ou les loisirs. De même, les trois postes de base considérés dans I'indice SFR comprennent les dépenses de luxe des ménages favorisés, ce qui affecte à la hausse les cœfficients moyens d'effort des ménages pour les besoins essentiels. Comme le seuil est calculé à partir de la consommation des ménages, seront considérés comme pauvres ceux qui consomment peu et épargnent beaucoup. Enfin, le choix des ménages de référence est problématique dans le calcul du SFR. Par exemple, les ménages jeunes se comparent-ils aux autres ménages jeunes, aux retraités ou à la moyenne de la population? Comment départager la référence aux normes de l'ensemble de la société et aux normes particulières du groupe d'appartenance?

La Mesure de faiblesse du revenu a été proposée par Statistique Canada à partir de 1990 afin de répondre à certaines critiques formulées à l'endroit du SFR. Cette seconde mesure définit la pauvreté comme étant la moitié de la médiane du revenu pondéré par unité. Ce seuil est calculé par Statistique Canada à partir du revenu brut et non plus des dépenses des ménages. Notons au passage qu'il serait préférable de le construire en prenant le revenu disponible, afin de tenir compte de l'effet redistributeur de l'impôt. Le problème du choix de la référence dans la construction du seuil MFR se pose avec plus d'acuité, car il est calculé sans tenir compte des différences importantes de niveaux de vie et de modes de vie entre les provinces et entre les villes. Ce seuil est comparable d'un pays à l'autre, ce qui explique qu'il est utilisé dans un grand nombre de pays développés.

II n'y a pas de seuil officiel de pauvreté au Canada, et Statistique Canada incite à ne pas considérer les deux mesures proposées comme des seuils de pauvreté, parce qu'elles ne sont pas construites à cet effet (Statistique Canada, 1995; Wolfson et Evans, 1990). Cette mise en garde n'est cependant pas respectée par plusieurs organismes - le Conseil national du bien-être social (1994), par exemple - et des chercheurs n'hésitent pas à utiliser ces seuils pour estimer l'incidence de la pauvreté ou pour I'analyser (Ross, 1984 ; Harp et Hofley, dir., 1971).

Le critère de mesure de la pauvreté définie en termes de privations et de conditions de vie a été également critiqué du fait des problèmes posés par la définition des dimensions des conditions de vie (qui changent dans le temps et selon les populations de référence) et par l'agrégation selon une hypothèse de séparabilité additive des 
différentes privations. La définition de la pauvreté par un seuil subjectif selon la méthode de van Praag (1977) pose enfin des problèmes d'estimation économétrique généralement négligés qui entraînent une sous-estimation de l'élasticité-revenu de ce seuil de pauvreté et une surestimation du nombre de pauvres.

Enfin, en plus d'estimer la proportion d'une population qui est pauvre, il paraît nécessaire de caractériser la gravité de la pauvreté. Le taux de pauvreté ou le comptage du nombre des pauvres ne renseignent pas sur l'ampleur de cette dernière, car même si le nombre de pauvres reste constant, l'état de ces derniers peut se détériorer par rapport à l'ensemble de la population non pauvre (Phipps, 1991). Les mesures du degré de pauvreté et de la perte de bien-être sont nombreuses, les plus connues étant sans doute les indices de Sen $(1976,1991)$, dont les propriétés sont discutées dans Foster et Shorrocks (1991), Zheng (1993) et Ravallion (1994).

\section{L'INDICE SYNTHÉTIQUE DE PAUVRETÉ-RICHESSE (ISPR)}

Afin de contourner les difficultés qu'entraîne la mesure de la pauvreté, nous proposons un nouvel indice synthétique de pauvreté-richesse (ISPR). Cet indice s'appuie sur une définition opératoire qui privilégie la prise en considération en même temps de trois dimensions différentes de la pauvreté : la non-satisfaction des besoins de base, la marginalisation par rapport à une population de référence du ménage et l'insuffisance du revenu relativement à l'ensemble de la société. Cet indice multidimensionnel tient compte du fait que la notion de pauvreté change avec la modification générale des conditions de vie: seront pauvres des ménages incapables de satisfaire leurs besoins fondamentaux, mais aussi ceux qui sont exclus d'une pleine participation à la vie en société.

L'indice ISPR tient également compte du caractère relatif de la pauvreté. Celle-ci est d'abord relative dans le temps, ce qui exige de prendre en considération l'augmentation de la richesse moyenne dans la société. Si la richesse est en hausse, il faut en tenir compte et élever le seuil qui définit la pauvreté. Mais la pauvreté est aussi relative à un groupe, comme l'ont bien montré le sociologue allemand Georg Simmel (1906) dans un article souvent cité et avant lui Alexis de Tocqueville dans son Mémoire sur le paupérisme (1835). On est pauvre par rapport aux normes acceptées dans l'ensemble de la société à un moment donné, certes, mais également par rapport aux normes du groupe social auquel on appartient ou auquel on s'identifie. Il importe donc de considérer ces deux aspects dans la 
construction de l'indice, et chacun des trois critères devra donc préciser en fonction de quelle référence il sera construit.

S'il est possible de définir de façon opératoire la pauvreté à partir d'un certain nombre de critères, ne serait-il pas aussi possible, et surtout fort pertinent pour l'analyse, de définir la richesse en adoptant une approche identique? Les seuils de pauvreté marquent un point sur un continuum en deçà duquel les ménages seront considérés comme pauvres; il serait normalement souhaitable de suivre la même logique pour établir, à l'autre bout du continuum, un seuil de richesse qui serait lui aussi à la fois relatif et absolu. Cette procédure n'a jamais été à notre connaissance adoptée dans les études sur la pauvreté, qui se contentent d'analyser la situation de dénuement dans la société. Or, l'approche qui est proposée ici permettra une analyse en parallèle de la pauvreté et de la richesse, ce dernier aspect n'étant cependant pas l'objet de la présente étude.

Voici, brièvement esquissée, la façon dont I'indice ISPR a été élaboré en tenant compte de la perspective qui vient d'être présentée.

a) Les ménages pauvres ont des dépenses relatives de subsistance plus élevées que celles de la population en général. Selon Townsend $(1979,1987)$, les ménages pauvres souffrent de privation parce qu'ils sont incapables de satisfaire leurs besoins de base. Depuis les travaux pionniers de Engel (1895) et de Halbwachs (1912), la part des dépenses totales consacrée à l'alimentation est un indicateur classique de subsistance et de privation. Nous proposons de définir comme pauvres selon ce premier critère les ménages qui consacrent, pour la proportion de leur budget qui va à l'alimentation au foyer, au moins un tiers de plus que les ménages de référence qu'on définira plus loin. Par ailleurs, seront considérés comme riches les ménages qui consacrent à se nourrir un tiers de leur budget de moins que la même population de référence.

b) Les seules dépenses de subsistance ne suffisent pas à définir la pauvreté dans les sociétés développées caractérisées par une extension considérable de la consommation marchande à toutes les sphères d'activité des ménages. Il faut aussi tenir compte de la non-satisfaction des besoins sociaux qui vont bien au-delà de la subsistance définie au sens strict. Paugam (1994) propose l'expression "pauvreté disqualifiante " pour qualifier en fait une forme d'exclusion sociale. Du point de vue sociologique, les ménages pauvres ne sont pas seulement incapables de subvenir adéquatement à leurs besoins de base, ils sont aussi des ménages marginalisés ou exclus qui ne parviennent pas à combler un minimum de besoins susceptibles $d$ 'assurer une pleine participation à la vie en société. Ces besoins sont maintenant très 
variés et, pour les satisfaire, les ménages doivent effectuer des dépenses aussi bien pour les communications ou les transports que pour les loisirs ou la santé. De ce point de vue, la pauvreté se mesure plutôt selon les conditions de vie. Il serait sans doute possible de définir un panier de base de biens et services nécessaires au bon fonctionnement en société, comme ont proposé de le faire Fugère et Lanctôt (1985) pour le Québec, par exemple. L'entreprise est cependant complexe et relativement arbitraire, sans oublier qu'elle repose sur le jugement porté par des experts.

Plutôt que d'identifier un panier de base, nous proposons de considérer comme pauvres les ménages qui dépensent moins de $67 \%$ du total des dépenses observées dans la population de référence et comme riches, ceux qui dépensent plus de $50 \%$. Les dépenses totales sont pondérées par le nombre de membres dans l'unité de consommation. Considérer les comportements de consommation - et non seulement le revenu, comme c'est le cas dans plusieurs indices de pauvreté - présente l'avantage de caractériser le niveau de vie réel des ménages en tenant compte des ressources obtenues par le travail au noir ou encore par les transferts privés en argent qui sont non négligeables dans bien des cas. La prise en compte de cette dimension permet d'éviter ainsi de considérer comme pauvres des ménages ayant des ressources cachées ou peu de revenus déclarés. Une étude récente de Brown (1994) a montré que, aux États-Unis, le portrait de la pauvreté dans les années 80 était différent (moins grave, en fait) lorsqu'on mesurait cette dernière à partir des dépenses plutôt qu'à partir des seuls revenus. Cela est dû probablement au fait que la dépense totale intègre des revenus de transfert, en particulier les transferts intergénérationnels, mais également sans doute au fait que la dépense totale est lissée par rapport au revenu courant et qu'elle constitue un bon indicateur du revenu permanent des ménages.

c) Par ailleurs, I'insuffisance du revenu est une source notable de pauvreté. Aussi le fait de considérer le revenu par unité de consommation comme troisième critère - et non seulement les dépenses totales par unité - $s$ 'avère-t-il essentiel parce que certains ménages peuvent avoir une consommation nettement inférieure à celle de leur groupe de référence, pour diverses raisons. On aurait tort de définir ces ménages comme pauvres, alors qu'en réalité ils consomment moins tout en ayant des revenus suffisants pour leur assurer éventuellement un niveau de vie décent. En conséquence, il faudrait caractériser les populations pauvres par une insuffisance de leur revenu permanent; cela consisterait cependant à supposer que les pauvres ne sont pas soumis à des contraintes de liquidités et qu'ils ont la possibilité d'effectuer des transferts intertemporels de leur 
richesse permanente, hypothèse assez discutable. À cette raison s'ajoute le fait qu'il est difficile de mesurer le revenu permanent dans les enquêtes, ce qui justifie d'utiliser l'insuffisance du revenu courant comme troisième critère de pauvreté. $D^{\prime}$ après ce dernier, seront pauvres les ménages qui se situent dans le quartile inférieur sur l'échelle des revenus disponibles par unité de consommation. Ce critère se distingue des deux précédents en ce qu'il situe cette fois les pauvres par rapport à toute la population dans la société. Le critère du revenu disponible par unité tient compte du caractère progressif de l'impôt sur le revenu, de l'ensemble des ressources pécuniaires courantes des ménages et de leur taille.

La pauvreté ne peut pas être mesurée seulement de manière absolue, puisqu'elle est aussi relative. Mais relative à quoi ? Ici deux pôles de référence s'imposent. Le premier est constitué par l'ensemble de la société. Nous posons que les ménages évalueront leur situation par rapport aux ressources qui sont disponibles à un moment donné dans leur société. Aussi le revenu des pauvres et des riches sera-t-il mesuré par référence à la distribution caractérisant l'ensemble de tous les ménages. Mais il existe un second pôle de référence qui est probablement à l'œuvre en même temps: on est pauvre dans sa classe sociale, comme l'a montré Simmel (1906), ou encore dans le groupe significatif auquel on s'identifie. Plus largement, les membres d'un ménage vont s'estimer pauvres par rapport à d'autres qui partagent les mêmes caractéristiques. Les pêcheurs de la Gaspésie ne se comparent pas nécessairement aux ouvriers de Montréal et les besoins subjectivement définis ne sont sans doute pas les mêmes dans les deux communautés. Il en va de même pour les jeunes - qui se comparent aux autres jeunes plutôt qu'aux adultes d'âge mûr -, tout comme pour les retraités qui évalueront leur situation par rapport à d'autres retraités. En conséquence, les dépenses totales et la part du budget consacrée à l'alimentation par chaque ménage seront analysées par rapport à un sous-groupe de référence et non pas par rapport à I'ensemble de la population comme c'est le cas pour le revenu disponible. L'indice ISPR tient compte de cette double référence: la société dans son ensemble et le sous-groupe auquel on s'identifie.

Comment définir le sous-groupe de référence? Ce dernier sera spécifié à partir de trois critères: le milieu de vie - ici la province ou la région au Canada -, la cohorte et le niveau de scolarité. Ces trois critères de référence sont quasiment invariants dans le temps: seul le critère d'appartenance à une cohorte d'âge est totalement invariant, mais les deux autres se modifient relativement peu sur le cycle de vie de la plupart des ménages. Lorsqu'un ménage change de région, on peut considérer que son adaptation aux conditions de vie de la 
nouvelle région est relativement rapide et que cette dernière constituera rapidement sa nouvelle référence. Le groupement des ménages par cohorte d'âge permet de neutraliser la déformation de la structure démographique d'une génération à l'autre. Les sous-populations que ces trois critères définissent sont donc assez stables et de taille assez importante pour pouvoir être considérées, sur le plan statistique, comme référence du ménage.

Doit-on prendre la moyenne ou la médiane? II est courant d'utiliser la médiane pour situer la position relative des ménages dans leur population de référence, afin d'éviter en particulier l'erreur de mesure de la moyenne. Néanmoins, les très bas et très hauts revenus constituent une information importante des ménages dans leur positionnement relatif, ce qui nous a amenés à les classer plutôt par rapport à la moyenne de leur population de référence. Pour tenir compte de l'erreur de mesure, on a défini un intervalle de confiance autour de la moyenne de classe $\mu$ à l'aide de l'écart type $\sigma$ et de l'effectif $\mathrm{n}$ de cette classe, selon l'expression

$$
\mu_{C} \pm t_{\alpha} \frac{O_{C}}{\sqrt{n_{C}}}
$$

dans laquelle le $t$ de Student est au seuil de risque $\alpha$ de $5 \%$. Les seuils de pauvreté et de richesse sur les deux premiers critères - le cœfficient budgétaire pour l'alimentation au foyer et les dépenses totales par unité de consommation - seront définis par rapport aux deux bornes (inférieure et supérieure) de cet intervalle et non pas par rapport à la seule moyenne. À noter au passage que le seuil de faible revenu (SFR) de Statistique Canada ne tient pas compte de l'erreur de mesure, ce qui est une faiblesse sur le plan statistique.

En résumé, seront caractérisés comme pauvres les ménages qui consacrent une partie de leur budget à la fonction alimentation au foyer qui est au moins un tiers plus élevée que celle observée dans les ménages de référence, qui dépensent moins de $67 \%$ du total observé dans ce groupe et qui se situent dans le quartile inférieur sur l'échelle de distribution des revenus disponibles par unité cette fois dans l'ensemble de la société. Les ménages riches seront ceux dont la part de leur budget consacrée à l'alimentation à domicile est inférieure ou égale à $67 \%$ de celle observée dans leur groupe de référence, qui dépensent plus de $50 \%$ du total des dépenses pondérées par unité observées dans leur groupe de référence et qui se situent dans le quartile supérieur de l'échelle des revenus disponibles par unité.

Nous noterons 1 le fait d'avoir un critère qui définit la pauvreté et 3 le fait d'avoir un critère de richesse, le résidu étant noté 2 . 
En d'autres termes, les ménages classés comme pauvres satisfont aux trois critères et les ménages quasi pauvres à deux critères sur trois. Les ménages riches auront quant à eux trois critères de richesse et les ménages quasi riches, deux critères. Au total, I'indice ISPR comprend 27 classes, qui ont été regroupées en cinq aux fins de l'analyse. Les cinq types retenus sont les suivants: pauvres (111), quasi pauvres $(112,121,211)$, quasi riches $(332,323,233)$ et riches $(333)$, alors que la classe moyenne regroupe tous les autres types. Nous ne diviserons pas ces derniers en sous-groupes, puisque nous nous intéressons en priorité ici à la mesure et à l'évolution de la pauvreté.

Une dernière précision s'impose. Atkinson (1987) a montré l'extrême variabilité du nombre de pauvres estimé à partir de diverses définitions des dépenses totales ou des revenus. On peut donc avancer qu'aucun de ces indicateurs classiques, pas plus que I'ISPR, ne permet un comptage robuste du nombre de pauvres. L'indice ISPR ne vise pas en priorité à estimer un taux de pauvreté, mais plutôt à obtenir une définition objective et opératoire de sous-populations sur lesquelles on pourra entreprendre des analyses microsociologiques des phénomènes de pauvreté et de richesse et sur lesquelles on pourra aussi effectuer des analyses fiables d'évolutions temporelles. Cet indice multidimensionnel permet de mesurer le degré d'appartenance des ménages à une classe socio-économique, mais il ne définit pas directement un seuil de pauvreté en termes de revenu. Cependant, il est possible d'estimer un tel seuil à partir de cet indice ISPR, en mesurant le revenu du dernier ménage classé dans la zone de pauvreté ou, de manière plus rigoureuse, le revenu du ménage situé au $95^{\mathrm{e}}$ centile de la classe de pauvreté pour éviter les erreurs de classement des ménages. Il pourrait donc y avoir des ménages non classés comme pauvres dans notre approche multidimensionnelle, mais dont le revenu serait inférieur à ce seuil. Ces ménages seraient pauvres selon le revenu, mais non pauvres selon les deux autres critères. Nous n'adoptons pas cette approche ici, car nous préférons caractériser la pauvreté à partir de trois dimensions plutôt qu'à partir du seul revenu.

Par ailleurs, l'intérêt de cet indice est de permettre d'effectuer des analyses longitudinales de sous-populations distinguées selon plusieurs critères et définis de la même manière dans des enquêtes répétées. II est possible d'étudier les tendances en évitant les biais systématiques qui apparaissent lorsqu'on utilise des critères de pauvreté qui ne tiennent pas compte des déformations sociodémographiques structurelles. L'indice ISPR est un instrument de mesure et $d^{\prime}$ analyse d'une portée générale qui sera utile notamment pour entreprendre des comparaisons internationales et des comparaisons dans 
le temps. Le fait de pouvoir reproduire une mesure fiable dans $d^{\prime}$ autres contextes nationaux à partir d'enquêtes faites selon des méthodologies proches présente un avantage non négligeable. Une étude sur la pauvreté en France suivant cette approche a été publiée par l'un des deux coauteurs de cet article (Cardoso et Gardes, 1996).

\section{LES DONNÉES}

Les premières mesures systématiques de la pauvreté ont été effectuées à partir des enquêtes budgétaires auprès des familles et des ménages. Nous adoptons ici la même approche. Les données qui seront analysées proviennent des enquêtes sur les dépenses des familles de Statistique Canada administrées en 1969, 1978, 1982, 1986 et 1992. L'analyse portera sur l'échantillon québécois seulement, mais nous proposerons une comparaison avec les autres régions du Canada afin de voir si la pauvreté est plus marquée au Québec qu'ailleurs. Nous avons effectué un certain filtrage des ménages interrogés en éliminant ceux qui avaient une dépense totale négative et ceux qui avaient aussi une dépense négative pour le poste automobile, de même que les dossiers voilés sur la variable province de résidence dans les trois dernières enquêtes. II s'agit en fait $d^{\prime}$ un nombre de cas très minime qui n'entache pas la représentativité des échantillons. Au total, 2957 ménages ont été retenus dans l'échantillon de 1969, 1868 en 1978, 2146 en 1982, 1973 en 1986 et 1870 en 1992.

Le tableau 1 présente l'évolution de la moyenne des principaux indicateurs utilisés dans la construction de l'ISPR. On voit l'importance de considérer séparément les revenus bruts et les revenus disponibles par unité de consommation, de même que les dépenses par unité, qui augmentent dans des proportions inégales. On notera au passage que les revenus réels moyens des ménages en dollars constants ont diminué entre 1978 et 1992, alors que le revenu total disponible par unité n'a pas suivi la même tendance. La baisse de la fécondité explique en partie cette observation, puisque la taille moyenne des ménages a diminué fortement. II faut cependant ajouter que la hausse du divorce a contribué, au cours de cette période, à créer plus de ménages ayant des revenus faibles ${ }^{2}$. II n'est donc pas neutre sur le plan méthodologique de prendre le revenu brut, le revenu disponible par unité de consommation ou encore la dépense totale par unité dans la construction d'une mesure de la pauvreté. En adoptant une approche multidimensionnelle, nous contournons la difficulté posée par l'existence de plusieurs facteurs susceptibles de causer la pauvreté. 
TABleau 1

Caractérisation de la population à l'aide de sept variables, selon l'année, 1969-1992, Québec

\begin{tabular}{lrrrrr}
\hline Variables & $\mathbf{1 9 6 9}$ & $\mathbf{1 9 7 8}$ & $\mathbf{1 9 8 2}$ & $\mathbf{1 9 8 6}$ & $\mathbf{1 9 9 2}$ \\
\hline $\begin{array}{l}\text { Revenu total brut } \\
\text { (\$1992) }\end{array}$ & 27125 & 45333 & 43311 & 42810 & 42124 \\
$\begin{array}{l}\text { Revenu disponible } \\
\text { par u.c. (\$1992) }\end{array}$ & 14753 & 16613 & 16497 & 16535 & 16578 \\
$\begin{array}{l}\text { Dépenses totales } \\
\text { par u.c. (\$ 1992) }\end{array}$ & 14853 & 16291 & 15651 & 16892 & 16432 \\
$\begin{array}{l}\text { Cœfficient budgétaire } \\
\text { alimentation }\end{array}$ & 0,288 & 0,197 & 0,181 & 0,165 & 0,157 \\
$\begin{array}{l}\text { Taille moyenne } \\
\text { du ménage }\end{array}$ & 3,82 & 3,09 & 2,89 & 2,72 & 2,56 \\
\% des 29 ans et moins & 15,5 & 21,1 & 17,8 & 16,1 & 11,4 \\
\% des 30-44 ans & 34,7 & 34,9 & 36,6 & 37,6 & 37,0 \\
\% des 45-64 ans & 35,5 & 31,4 & 31,9 & 30,4 & 34,0 \\
\% des 65 ans et plus & 14,3 & 12,5 & 13,7 & 15,9 & 17,6 \\
\% primaire & 48,0 & 32,8 & 30,7 & 28,2 & 25,3 \\
\% secondaire & 36,8 & 40,0 & 38,7 & 40,2 & 37,7 \\
\% post-secondaire & 15,3 & 27,2 & 31,3 & 31,6 & 36,8 \\
Taille de l'échantillon & 2957 & 1868 & 2146 & 1973 & 1870 \\
\hline
\end{tabular}

Source : Statistique Canada, Microdonnées des Enquêtes sur les dépenses des familles, calculs des auteurs.

Le cœfficient budgétaire pour la fonction alimentation au foyer marque une diminution régulière et constante sur toute la période. Cet indicateur privilégié indique bien une hausse du niveau de vie et l'émergence $d^{\prime} u n$ revenu discrétionnaire de plus en plus important. Ce critère montre ainsi les limites d'une approche qui privilégie uniquement le revenu pour caractériser la pauvreté. Même si le revenu total brut baisse quelque peu et même si le revenu disponible par habitant augmente très faiblement, les ménages peuvent dégager une marge de manœuvre et accroître leur revenu discrétionnaire.

La morphologie des ménages a aussi profondément changé depuis trente ans. Ainsi la proportion des ménages dont la personne de référence est âgée de plus de 65 ans s'accroît, alors que régresse celle des jeunes, et la proportion des ménages plus scolarisés a augmenté de façon marquée entre 1969 et 1992. Ce dernier facteur est aussi important, car il influence la perception qu'auront les membres du ménage de leur niveau de vie, toujours d'après la théorie de Tocqueville et de Simmel. Il est nécessaire de tenir compte de ces 
changements structuraux, non seulement dans la définition de la pauvreté, mais surtout dans l'analyse des évolutions temporelles de cette dernière.

\section{Évolution de la pauvreté et de la richesse des ménages}

Quel est le niveau de pauvreté estimé par l'indice ISPR? Comment se comparent les résultats obtenus avec ceux de Statistique Canada? L'intérêt de l'indice synthétique de pauvreté-richesse n'est pas d'abord de compter les ménages pauvres ou riches; il sert plutôt à identifier quels ménages sont pauvres dans une société donnée, afin d'analyser leurs caractéristiques ou encore de mesurer l'évolution de leur proportion dans le temps à partir d'une mesure opératoire fiable. Il distingue aussi les ménages pauvres selon qu'ils possèdent tous les attributs (entendus ici au sens méthodologique) de la pauvreté ou seulement quelques-uns. Enfin, les indices de Statistique Canada distinguent les ménages ne comptant qu'une seule personne et ceux qui en comptent deux ou plus, alors que I'ISPR prend en considération tous les ménages en pondérant leur composition. Ayant en tête ces différences opératoires, on peut additionner les proportions observées pour les deux groupes de pauvres définis selon I'indice ISPR et les comparer avec les proportions obtenues par Statistique Canada (tableau 2). L'indice ISPR (pauvres et quasi-pauvres confondus) donne une estimation quelque peu inférieure au Seuil de faible revenu en 1969, exactement la même estimation en 1978 en comparant avec le seuil révisé et une estimation supérieure à partir de 1986. Cet indice est par ailleurs supérieur au seuil MFR pour les trois années disponibles 1982, 1986 et 1992. On peut avancer que le Seuil de faible revenu de Statistique Canada et I'ISPR corrèlent très bien pour les années 1969 à 1982. Après cette date, le seuil de Statistique Canada aurait tendance à sous-estimer la pauvreté, parce qu'il ne mesure pas aussi bien la hausse de la quasi-pauvreté, c'est-à-dire la hausse du nombre des ménages n'ayant que certains attributs de la pauvreté. Cette analyse donne à penser que l'indice ISPR a une bonne validité externe. Par ailleurs, il apparaît plus précis que les deux mesures de Statistique Canada, notamment parce qu'il est mieux en mesure de bien caractériser l'évolution fort différenciée de la quasipauvreté sur une longue période.

Les tendances d'évolution de l'indice ISPR sont les suivantes. Nous analyserons d'abord l'évolution de la proportion des ménages ayant trois critères de pauvreté. On observe une certaine augmentation de la proportion des ménages pauvres jusqu'au milieu des années 80 , suivie d'une réduction en 1992, dernière année 
TABLEAU 2

Indices mesurant la pauvreté (SFR, MFR et ISPR) selon l'année 1969-1992, Québec

\begin{tabular}{llccrrr}
\hline Indice & & $\mathbf{1 9 6 9}$ & $\mathbf{1 9 7 8}$ & $\mathbf{1 9 8 2}$ & $\mathbf{1 9 8 6}$ & $\mathbf{1 9 9 2}$ \\
\hline \multirow{2}{*}{ SFR $^{*}$} & Base 1969 & 24,2 & 12,0 & 12,0 & 10,5 & - \\
& Base 1978 & & $14,6^{* *}$ & 15,0 & 14,6 & \\
& Base 1986 & - & 15,3 & 16,7 & 17,1 & 15,5 \\
& Base 1992 & - & - & 16,7 & 16,7 & 15,3 \\
MFR $^{*}$ & & - & - & 14,8 & 14,3 & 12,7 \\
ISPR & Pauvres & 5,7 & 6,4 & 7,1 & 8,0 & 6,4 \\
& Quasi-pauvres & 15,6 & 8,2 & 9,7 & 9,6 & 10,6 \\
& Total & 21,3 & 14,6 & 16,8 & 17,6 & 17,0 \\
\hline
\end{tabular}

Ménages de 2 personnes et plus

** 1979

Source : Statistique Canada, cat. 13-207 ; microdonnées des Enquêtes sur les dépenses des familles, calculs des auteurs. Les données sur les MFR et les SFR pour les années 1986 et 1992 ont été révisées par Statistique Canada en 1996, ce qui explique l'existence de certains écarts avec les publications précédentes.

disponible (tableau 2). Ce résultat est quelque peu surprenant, car il va à l'encontre de l'image répandue d'une réduction importante de la pauvreté durant les années 70 . En fait, I'indice ISPR montre que le noyau dur de la pauvreté - la proportion de ménages qui sont les plus démunis selon les trois critères proposés - n'a pas beaucoup bougé en termes statistiques depuis plus de trente ans, et la tendance à long terme serait même à une légère augmentation. L'estimation de la proportion des ménages pauvres se rapproche de celle faite par Sarlo (1992) qui se fonde sur la non-satisfaction des besoins de base des ménages.

Il en va autrement pour la proportion des ménages qui sont quasi pauvres, c'est-à-dire ceux qui ont deux critètes de pauvreté sur trois d'après notre approche. La proportion de ces quasi-pauvres a régressé de façon importante au cours des années 70, baissant presque de moitié en dix ans. Par la suite, la proportion des quasipauvres a augmenté de façon continue durant les années 80 et 90 . Ce résultat est important, car il permet une analyse plus fine de l'évolution de la pauvreté sur une période de 25 ans.

Comment interpréter les évolutions divergentes des deux groupes de pauvres et quasi-pauvres? Les programmes sociaux du début des années 70 ont fait régresser la pauvreté, sans oublier l'impact du travail salarié des épouses dans les ménages et la baisse de la 
fécondité des couples. Une proportion grandissante des ménages possèdent maintenant quelques attributs de la population pauvre, dont les caractéristiques sont par ailleurs plus diverses qu'auparavant. Cette augmentation est sans doute due à la fois aux mutations du marché du travail (hausse du chômage et de la précarité, baisse des salaires relatifs, etc.) et aux mutations observables dans les modes de vie (hausse du divorce et augmentation de la monoparentalité, etc.). Ce résultat confirme que nous sommes en présence $d^{\prime}$ une aggravation de la perte de bien-être - au sens de welfare loss de Sen, comme I'a montré l'étude de Phipps (1991) pour le Canada. Cette évolution différente souligne une fois de plus la nécessité ou l'intérêt de distinguer conceptuellement et opératoirement différents types de pauvreté (Hanratty et Blank, 1992).

La classe moyenne a connu un gonflement important au cours des années 70 , suivi d'un léger déclin au début des années 80 , déclin cependant beaucoup moins prononcé que celui qui est mesuré par un indice de polarisation des revenus individuels (Wolfson, 1995; Morissette, Myles et Picot, 1994). On ne peut pas soutenir que la classe moyenne ait été en net déclin au cours des années 80 d'après I'indice ISPR qui est construit à partir du revenu familial disponible par unité de consommation. Une étude récente effectuée par une économiste du Mouvement Desjardins sur les inégalités va dans le même sens (Bégin, 1996). Deux facteurs au moins ont freiné le déclin de la situation socio-économique des familles et des ménages de classe moyenne, alors que la position des individus subissait de fortes

TABLEAU 3

Répartition de la population des ménages d'après l'indice synthétique de pauvreté-richesse (ISPR), 1969-1992, Québec

\begin{tabular}{lrrrrr}
\hline Indice ISPR & $\mathbf{1 9 6 9}$ & $\mathbf{1 9 7 8}$ & $\mathbf{1 9 8 2}$ & $\mathbf{1 9 8 6}$ & $\mathbf{1 9 9 2}$ \\
\hline Pauvres & 5,7 & 6,4 & 7,1 & 8,0 & 6,4 \\
Quasi-pauvres & 15,6 & 8,2 & 9,7 & 9,6 & 10,6 \\
Classe moyenne & 64,8 & 71,2 & 68,5 & 68,6 & 69,1 \\
Quasi-riches & 9,7 & 9,0 & 9,3 & 8,7 & 8,9 \\
Riches & 4,2 & 5,2 & 5,3 & 5,1 & 5,0 \\
Total \% & 100 & 100 & 100 & 100 & 100 \\
Nombre & 2957 & 1868 & 2146 & 1973 & 1870 \\
\hline
\end{tabular}

Source: Statistique Canada, microdonnées des Enquêtes sur les dépenses des familles, calculs des auteurs. 
pressions vers le bas: la participation accrue au marché du travail des femmes mariées avec enfants et, ensuite, la diminution importante de la fécondité des couples. Ce déclin autrement aurait été marqué compte tenu de l'évolution des revenus individuels (Langlois, 1994). On retiendra cependant que la forte croissance de la proportion des ménages se retrouvant au centre, qui avait été observée durant les années $70, s^{\prime}$ est brusquement arrêtée, alors que s'amorce après cette date une certaine décroissance, encore faible mais très perceptible. La baisse de la fécondité et le travail salarié des épouses dans les familles ont sans doute réussi à freiner pendant une quinzaine d'années le déclin des familles appartenant à la classe moyenne. Celles-ci ne pourront cependant plus compter sur ces deux facteurs pour maintenir leur position socio-économique durant les années 90 , et il est permis de penser que la situation de plusieurs d'entre elles risque de se détériorer.

À l'autre bout de l'échelle, la proportion des ménages quasi riches a diminué au cours des années 70 , alors que celle des riches augmentait quelque peu, mais les évolutions observables par la suite dans ces deux catégories ont été moins marquées que celles qui caractérisent les ménages pauvres.

Que retenir de ces évolutions? Nous retrouvons ici un résultat important: on observe l'amorce d'un glissement vers le bas d'une fraction de la classe moyenne - fraction encore minime il est vrai, mais la tendance est là -, alors qu'augmente la proportion des ménages que nous avons appelés les quasi-pauvres. Ces derniers ménages ne vivent pas dans le dénuement ou la privation complète, comme $c^{\prime}$ est le cas pour les pauvres, mais ils sont par ailleurs exclus du rêve de faire partie de la classe moyenne. C'est ce glissement vers le bas qui alimente la croissance de la quasi-pauvreté qui s'est amorcée vers le milieu des années 70 .

\section{Pauvreté, effet de génération et capital scolaire}

Nous analyserons maintenant l'évolution de I'indice ISPR par groupes d'âge. La situation des ménages change de façon assez radicale, entre 1969 et 1978, dans le contexte d'une forte croissance économique et à la suite de l'avènement de l'État-providence. La pauvreté est en régression chez les personnes les plus âgées et en nette augmentation chez les jeunes ménages depuis cette date. Ce résultat confirme ce que plusieurs recherches ont observé en comparant des données transversales sans correction de l'effet cycle de vie ni de l'effet de la structure d'âge qui n'est pas la même durant toute la période (MMSR, 1990 ; Langlois, 1990 ; Zouali, 1993; Gauthier et Mercier, 1994). 
La quasi-pauvreté reste importante chez les personnes âgées. Les divers programmes de soutien du revenu mis en place par l'État en leur faveur ont réussi à faire diminuer de façon marquée la pauvreté, mais non la quasi-pauvreté telle que définie ici. Il faut noter une nette tendance à la moyennisation - pour reprendre un concept proposé par Henri Mendras (1990) - chez les personnes âgées : on trouve dans ce groupe moins de pauvres et aussi moins de riches, la plupart des ménages formés de personnes âgées se concentrant au centre.

Mis à part ce groupe des personnes âgées, la probabilité de faire partie de la classe moyenne $\mathrm{n}^{\prime}$ a pas vraiment changé entre 1992 et 1969, comme on peut le voir dans le tableau 4. On remarque un certain affaissement du centre vers le milieu des années 80 - période au cours de laquelle le discours sur le déclin de la classe moyenne est apparu -, mais la situation semble s'être corrigée quelque peu par la suite. En fait, $c^{\prime}$ est dans la dernière moitié de la vie active qu'on observe la probabilité la plus faible de faire partie du centre, alors qu'augmentent à la fois la proportion des riches et celle des pauvres. Tout se passe comme s'il y avait une plus grande polarisation à cette étape du cycle de vie.

La pauvreté régresse à mesure que s'élève le niveau d'éducation (tableau 5). La réduction de la pauvreté entre le primaire et le secondaire est plus faible qu'entre les niveaux secondaire et postsecondaire. Le fait de poursuivre des études postsecondaires est une sorte d'assurance contre la pauvreté, mais il n'est pas une garantie d'accès à la richesse. Sur le plan temporel, la proportion de ménages pauvres diminue entre 1978 et 1992 chez les diplômés du primaire, mais non chez ceux du secondaire et du postsecondaire, deux catégories dans lesquelles on note une même augmentation de la proportion des ménages pauvres après 1982. La hausse de la proportion des ménages quasi pauvres est plus marquée chez les diplômés du postsecondaire après 1978 . Au total, le diplôme s'avérait plus rentable durant les années 60, mais il l'est moins durant les années 80.

\section{Plus de pauvres au Québec?}

La mesure de la pauvreté a d'importantes répercussions politiques, puisqu'elle sert en quelque sorte d'aulne pour porter un jugement sur le progrès social ou pour critiquer l'orientation des politiques publiques. Certains voient dans l'entreprise de définir de nouveaux indices de pauvreté une tentative à peine déguisée pour chercher à diminuer l'importance du phénomène. Ce fut le cas pour le travail de Sarlo (1992), il y a quelques années. La comparaison des taux de 
TABleau 4

Indice synthétique pauvreté-richesse (ISPR) selon les groupes d'âge de la personne de référence et l'année, 1969-1992, Québec

\begin{tabular}{lrrrrrrr}
\hline Âge & Pauvres & $\begin{array}{c}\text { Quasi- } \\
\text { pauvres }\end{array}$ & $\begin{array}{c}\text { Classe } \\
\text { moyenne }\end{array}$ & $\begin{array}{r}\text { Quasi- } \\
\text { riches }\end{array}$ & Riches & Total \% & N \\
\hline 1969 & & & & & & & \\
Moins de 30 ans & 3,5 & 13,3 & 71,8 & 8,1 & 3,3 & 100 & 458 \\
30-44 & 4,5 & 13,3 & 67,6 & 10,6 & 4,0 & 100 & 1025 \\
45-64 & 5,9 & 14,3 & 63,6 & 11,3 & 4,9 & 100 & 1050 \\
65 et plus & 10,6 & 26,7 & 53,3 & 5,2 & 4,2 & 100 & 424 \\
1978 & & & & & & & \\
Moins de 30 ans & 6,3 & 5,6 & 72,9 & 10,1 & 5,1 & 100 & 395 \\
30-44 & 6,7 & 6,4 & 70,8 & 9,5 & 6,6 & 100 & 652 \\
45-64 & 6,8 & 9,9 & 69,0 & 9,5 & 4,8 & 100 & 587 \\
65 et plus & 4,3 & 13,7 & 74,3 & 4,7 & 3,0 & 100 & 234 \\
1982 & & & & & & & \\
Moins de 30 ans & 6,6 & 8,7 & 70,3 & 11,3 & 3,1 & 100 & 381 \\
30-44 & 8,0 & 9,3 & 65,0 & 10,2 & 7,5 & 100 & 786 \\
45-64 & 7,9 & 9,9 & 67,5 & 9,4 & 5,3 & 100 & 684 \\
65 et plus & 3,7 & 11,9 & 77,5 & 4,4 & 2,4 & 100 & 295 \\
1986 & & & & & & & \\
Moins de 30 ans & 10,4 & 9,5 & 67,8 & 9,1 & 3,2 & 100 & 317 \\
30-44 & 8,1 & 9,3 & 66,7 & 9,3 & 6,6 & 100 & 742 \\
45-64 & 6,7 & 10,2 & 69,1 & 9,3 & 4,7 & 100 & 600 \\
65 et plus & 7,6 & 9,6 & 72,9 & 5,7 & 4,1 & 100 & 314 \\
Moins de 30 ans & 5,2 & 8,8 & 72,8 & 9,4 & 3,8 & 100 & 213 \\
30-44 & 7,7 & 10,3 & 67,5 & 8,4 & 6,1 & 100 & 692 \\
65 et plus & 7,2 & 11,0 & 64,1 & 12,0 & 5,7 & 100 & 635 \\
\hline
\end{tabular}

Source : Statistique Canada, Microdonnées des Enquêtes sur les dépenses des familles, calculs des auteurs.

pauvreté entre provinces au sein du Canada, ou encore entre pays ayant des systèmes différents de protection sociale, a elle aussi des implications politiques, car elle est susceptible d'alimenter la recherche du meilleur système sociopolitique. 


\section{TABLEAU 5}

Indice synthétique pauvreté-richesse (ISPR) selon le niveau de scolarité de la personne de référence et l'année, 1969-1992, Québec

\begin{tabular}{|c|c|c|c|c|c|c|c|}
\hline Scolarité & Pauvres & $\begin{array}{c}\text { Quasi- } \\
\text { pauvres }\end{array}$ & $\begin{array}{c}\text { Classe } \\
\text { moyenne }\end{array}$ & $\begin{array}{l}\text { Quasi- } \\
\text { riches }\end{array}$ & Riches & Total \% & $N$ \\
\hline \multicolumn{8}{|l|}{1969} \\
\hline Primaire & 7,3 & 19,8 & 60,2 & 8,5 & 4,2 & 100 & 1419 \\
\hline Secondaire & 5,5 & 12,0 & 66,0 & 9,9 & 4,6 & 100 & 1087 \\
\hline Postsecondaire & 1,1 & 10,9 & 71,6 & 12,9 & 3,5 & 100 & 451 \\
\hline \multicolumn{8}{|l|}{1978} \\
\hline Primaire & 8,3 & 12,6 & 67,8 & 7,0 & 4,2 & 100 & 612 \\
\hline Secondaire & 7,4 & 7,5 & 71,0 & 8,6 & 5,6 & 100 & 748 \\
\hline Postsecondaire & 2,6 & 4,1 & 75,2 & 12,2 & 5,9 & 100 & 508 \\
\hline \multicolumn{8}{|l|}{1982} \\
\hline Primaire & 8,1 & 13,2 & 69,1 & 7,0 & 2,6 & 100 & 644 \\
\hline Secondaire & 9,3 & 9,3 & 67,0 & 7,9 & 6,5 & 100 & 831 \\
\hline Postsecondaire & 3,6 & 7,0 & 69,7 & 13,3 & 6,4 & 100 & 671 \\
\hline \multicolumn{8}{|l|}{1986} \\
\hline Primaire & 8,3 & 13,3 & 69,4 & 5,2 & 3,8 & 100 & 556 \\
\hline Secondaire & 9,7 & 9,7 & 66,1 & 8,7 & 5,8 & 100 & 793 \\
\hline Postsecondaire & 5,4 & 6,3 & 71,2 & 11,9 & 5,3 & 100 & 624 \\
\hline \multicolumn{8}{|l|}{1992} \\
\hline Primaire & 6,8 & 13,7 & 69,8 & 6,6 & 3,2 & 100 & 474 \\
\hline Secondaire & 9,2 & 10,4 & 67,4 & 6,8 & 6,2 & 100 & 707 \\
\hline Postsecondaire & 3,2 & 8,7 & 70,5 & 12,6 & 4,9 & 100 & 689 \\
\hline
\end{tabular}

Source : Statistique Canada, Microdonnées des Enquêtes sur les dépenses des familles, calculs des auteurs.

Y a-t-il plus de pauvres au Québec que dans les autres régions du Canada? Les indicateurs existants ont amené les chercheurs à donner une réponse positive à cette question, qui a par ailleurs fait l'objet de vifs débats dans le grand public. L'indicateur ISPR arrive-t-il aux mêmes conclusions? L'écart entre le Québec et l'Ontario a-t-il bien tendance à s'élargir, comme l'ont montré les études basées sur les mesures proposées par Statistique Canada? L'indice ISPR s'avérera précieux pour répondre à ces questions, car l'un des critères de validité d'une mesure est de voir comment elle 
$\mathrm{s}^{\prime}$ harmonise avec d'autres mesures susceptibles de caractériser le même univers de contenu.

Pour comparer le Québec avec les autres provinces, il paraît nécessaire de rappeler quelles sont les données de Statistique Canada qui fondent les analyses comparées existantes. Le tableau 6 présente les seuils SFR, MFR et ISPR pour cinq grandes régions: les Provinces de l'Atlantique, le Québec, l'Ontario, les Prairies et la ColombieBritannique. L'indice SFR - et surtout l'indice MFR - donnent des écarts très marqués entre les grandes régions du Canada. On peut faire deux lectures de ces écarts du point de vue de la validité des mesures. $D^{\prime}$ une part, ces différences peuvent correspondre à la réalité - et en ce cas il y aurait effectivement plus de pauvres dans les régions périphériques et au Québec -, mais il se peut aussi que les mesures actuelles sous-estiment la pauvreté dans les provinces riches et la surestiment dans les autres provinces, ce qui accentuerait les écarts, et en ce cas la mesure serait biaisée. Nous avons montré plus haut qu'il y avait de sérieuses raisons de penser que la façon de calculer les seuils chez Statistique Canada contribuait à accentuer les écarts entre provinces riches et provinces pauvres. L'indice ISPR clarifie les choses en précisant la référence des ménages - référence qui est la communauté d'appartenance (province et groupe d'âge), mais aussi la société dans son ensemble qui détermine une norme nationale - et en proposant une approche multidimensionnelle de la pauvreté.

Que donne la comparaison des indices ISPR ? C'est dans les Prairies que le taux de pauvreté est le plus faible en 1992, suivi par les Provinces de l'Atlantique. Par ailleurs, l'écart entre l'Ontario et le Québec est fortement réduit; les taux de pauvreté étaient presque identiques en Ontario et au Québec $(6,4 \%$ et $6,5 \%)$ en 1992, la Colombie-Britannique suivant derrière avec $6 \%$. Ces résultats sont assez différents de ceux obtenus avec les deux mesures de Statistique Canada. D'après I'ISPR, il y aurait moins de pauvres dans les Provinces de l'Atlantique et dans les Prairies, et plus dans les deux provinces les plus riches: I'Ontario et la Colombie-Britannique, de même qu'au Québec. Sur le plan longitudinal, le taux de pauvreté est en régression dans les Provinces de l'Atlantique, il est relativement stable dans les Prairies (avec un sommet en 1982), alors qu'il augmente en Ontario et en Colombie-Britannique.

Si nous additionnons les deux taux (de pauvreté et de quasipauvreté), on obtient des estimations généralement un peu plus élevées que les mesures de Statistique Canada pour caractériser la pauvreté dans son ensemble. Globalement, celle-ci progresse depuis 1978 dans les trois provinces les plus populeuses, soit au Québec, en 
TABLEAU 6

Taux de pauvreté selon différents indices - SFR, MFR et ISPR -, la région au Canada et l'année, 1969-1992

\begin{tabular}{|c|c|c|c|c|c|c|c|c|}
\hline \multirow[b]{2}{*}{$\begin{array}{l}\text { Région } \\
\text { Année }\end{array}$} & \multicolumn{4}{|c|}{ SFR" } & \multirow[t]{2}{*}{ MFR $^{* *}$} & \multicolumn{3}{|c|}{ SPR } \\
\hline & $\begin{array}{l}\text { Base } \\
1969 \\
\end{array}$ & $\begin{array}{l}\text { Base } \\
1978 \\
\end{array}$ & $\begin{array}{l}\text { Base } \\
1986 \\
\end{array}$ & $\begin{array}{l}\text { Base } \\
1992 \\
\end{array}$ & & Pauvres & $\begin{array}{l}\text { Quasi- } \\
\text { pauvres }\end{array}$ & Total \\
\hline \multicolumn{9}{|l|}{ Atlantique } \\
\hline 1969 & 33,0 & - & - & - & - & 7,1 & 14,6 & 21,7 \\
\hline 1978 & 14,5 & $17,2^{*}$ & - & - & - & 6,2 & 8,8 & 15,0 \\
\hline 1982 & 13,0 & 17,4 & 17,8 & 18,1 & 22,9 & 6,1 & 9,6 & 15,7 \\
\hline 1986 & 11,2 & 15,1 & 15,6 & 15,7 & 19,9 & 7,2 & 9,2 & 16,4 \\
\hline 1992 & - & - & 14,2 & 13,8 & 17,4 & 5,8 & 9,4 & 15,2 \\
\hline \multicolumn{9}{|l|}{ Québec } \\
\hline 1969 & 24,2 & - & - & - & - & 5,7 & 15,6 & 21,3 \\
\hline 1978 & 12,0 & $14,6^{*}$ & 15,3 & - & - & 6,4 & 8,2 & 14,6 \\
\hline 1982 & 12,0 & 15,0 & 16,7 & 16,7 & 14,8 & 7,1 & 9,7 & 16,8 \\
\hline 1986 & 10,5 & 14,6 & 17,1 & 16,7 & 14,3 & 8,0 & 9,6 & 17,6 \\
\hline 1992 & - & - & 15,5 & 15,9 & 12,7 & 6,4 & 10,6 & 17,0 \\
\hline \multicolumn{9}{|l|}{ Ontario } \\
\hline 1969 & 14,4 & - & - & & - & 5,7 & 13,6 & 19,3 \\
\hline 1978 & 8,8 & $11,4^{*}$ & - & & - & 5,0 & 9,2 & 14,2 \\
\hline 1982 & 8,7 & 10,9 & 12,1 & 12,2 & 8,3 & 6,1 & 8,6 & 14,7 \\
\hline 1986 & 6,7 & 8,5 & 10,1 & 9,8 & 8,5 & 6,1 & 10,0 & 16,1 \\
\hline 1992 & - & - & 11,2 & 11,1 & 10,2 & 6,5 & 9,6 & 16,1 \\
\hline \multicolumn{9}{|l|}{ Prairies } \\
\hline 1969 & 26,2 & - & - & - & - & 4,9 & 15,1 & 20,0 \\
\hline 1978 & 10,2 & $13,7^{*}$ & - & - & - & 5,6 & 9,9 & 15,5 \\
\hline 1982 & 9,1 & 10,8 & 11,8 & 11,8 & 14,9 & 6,8 & 10,6 & 17,4 \\
\hline 1986 & 9,2 & 12,0 & 14,1 & 13,8 & 13,3 & 5,8 & 10,4 & 16,2 \\
\hline 1992 & - & - & 15,2 & 15,2 & 15,6 & 5,0 & 9,3 & 14,3 \\
\hline \multicolumn{9}{|c|}{ Colombie-Britannique } \\
\hline 1969 & 16,7 & - & - & - & - & 4,6 & 12,9 & 17,5 \\
\hline 1978 & 8,3 & $11,3^{*}$ & - & - & - & 4,6 & 7,4 & 12,0 \\
\hline 1982 & 9,4 & 11,9 & 13,8 & 13,5 & 12,4 & 4,9 & 8,9 & 13,8 \\
\hline 1986 & 10,3 & 13,2 & 15,6 & 15,1 & 13,6 & 5,7 & 10,0 & 15,7 \\
\hline 1992 & - & - & 13,4 & 13,5 & 11,4 & 6,0 & 9,5 & 15,5 \\
\hline
\end{tabular}

Année 1979

* Ménages de deux personnes et plus et familles.

Source : Statistique Canada, cat. 13-207, microdonnées des Enquêtes sur les dépenses des familles, calculs des auteurs. Les données sur les MFR et les SFR pour les années 1986 et 1992 ont été révisées par Statistique Canada en 1996, ce qui explique l'existence de certains écarts avec les publications précédentes. 
Ontario et en Colombie-Britannique, elle est relativement stable dans les Provinces de l'Atlantique et elle régresse dans les Prairies. II faut souligner que l'écart avec les données fédérales est plus marqué dans le cas de l'Ontario, la province la plus riche.

Nous sommes maintenant en mesure de répondre à la question posée plus haut. Effectivement, si nous additionnons les deux taux distingués dans I'indice ISPR, C'est au Québec que la proportion de pauvres et de quasi-pauvres est la plus élevée : $17 \%$ en 1992, suivi de l'Ontario avec 16,1\%. Le diagnostic pessimiste posé sur le Québec n'est pas modifié, mais ce qui change, c'est l'écart qui sépare le Québec des autres provinces, écart qui se rétrécit de façon marquée, notamment l'écart avec l'Ontario, point de comparaison souvent privilégié.

\section{CONCLUSION}

L'indice synthétique de pauvreté-richesse (ISPR) que nous proposons caractérise la pauvreté plus précisément que les indices usuels de Statistique Canada, parce qu'il est multidimensionnel et qu'il tient compte de références plus précises. De plus, cet indice distingue également les ménages de classe moyenne des ménages de classes riches, ce qui permet de situer la place de la pauvreté dans I'ensemble de la société. D'après I'ISPR, la proportion de pauvres diminue au Québec dans les tranches d'âge élevées, alors qu'elle augmente chez les jeunes; aussi, la pauvreté apparaît en hausse chez les diplômés.

L'indice donne un portrait différent de la pauvreté dans l'ensemble du Canada - et notamment dans les provinces riches, là où les indices usuels de Statistique Canada semblent sous-estimer le phénomène - en montrant que la pauvreté (les deux types confondus) y est largement répandue et en croissance, notamment en Ontario et en Colombie-Britannique. L'analyse confirme l'observation qu'il y a plus de pauvres au Québec qu'ailleurs au Canada. II importe cependant de préciser que l'écart qui sépare le Québec et l'Ontario s'amenuise grandement, ce qui montre que le problème de la pauvreté n'est pas l'apanage des provinces périphériques ou du Québec. La pauvreté est un problème qui touche aussi les provinces les plus riches du Canada. 


\section{Notes}

1. Les auteurs remercient Patrice Gaubert (Lamia, Université de Paris-Sorbonne) et Johanne Bujold (INRS-Culture et société) pour leur aide à construire les fichiers de données, de même que le ministère de la Santé et des Services sociaux du Québec, qui a accordé une aide financière pour mener à terme cette recherche. Nous tenons aussi à exprimer notre gratitude aux collègues qui ont participé à différents séminaires tenus au département de sociologie et au département d'économique de l'Université Laval, au Lamia (Paris), au Credoc (Paris) et au Laboratoire de sociologie quantitative du CREST (INSEE, France). Enfin, nos remerciements vont à Statistique Canada, qui nous a donné accès à l'Enquête sur les dépenses des familles.

Les auteurs de l'article sont les seuls responsables de l'analyse des données. On est prié d'adresser toute correspondance à Simon Langlois, Département de sociologie, Université Laval, Québec G1K 7P4 (téléc. : 418 656-7390).

2. Lorsqu'un couple divorce, deux nouveaux ménages ayant des revenus totaux plus faibles remplacent le plus souvent le ménage d'origine, du moins pendant un certain temps.

\section{Références bibliographiques}

ATKINSON, A.B. (1987). "On the Measurement of Poverty », Econometrica, vol. $55, \mathrm{n}^{\circ} 4: 749-764$.

ALCOCK, P. (1993). Understanding Poverty, Chatham: Macmillan.

BÉGIN, H. (1996). "Hausse de l'inégalité des revenus au Québec: mythe ou réalités?", En perspectives. Mouvement Desjardins, vol. 6, février: 1-3.

BROWN, C. (1994). American Standard of Living, 1918-1988. Cambridge: Blackwell.

CAllan, T., B. NOLAN et C.T. WHELAN (1993). "Resources Deprivation and the Measurement of Poverty ", Journal of Social Policy, vol. 22, $\mathrm{n}^{\circ} 2$ : $141-172$.

CARDOSO, N. et F. GARDES (1996). "Caractérisation et analyse des comportements de consommation des ménages pauvres sur données individuelles françaises ", Revue économique : 687-698.

Citro, C.F. et R.T. MiChael (dir.) (1995). Measuring Poverty. A New Approach. Washington: National Academy Press.

CONSEIL ÉCONOMIQUE DU CANADA (1993). Les nouveaux visages de la pauvreté. La sécurité du revenu des familles canadiennes. Ottawa.

CONSEIL NATIONAL DU BIEN-ÊTRE SOCIAL (1994). Profil de la pauvreté. Ottawa.

ENGEL, E. (1895) . "Die Lebenskosten Belgischer Arbeiter-familien Früher UndJjetzt ", International Statistical Institute Bulletin, 9: 1-74.

FOSTER, J.E. et A.F. SHORROCKS (1991). "Subgroup Consistent Poverty Indices ", Econometrica, mai : 687-709.

Foster, J.E., F.J. GreER et E. ThORBECK (1984). "A Class of Decomposable Poverty Measures », Econometrica, mai : 761-765 
FUGÈRE, D. et P. LANCTÔT (1985). Méthodologie de détermination des seuils de revenu minimum au Québec. Québec: ministère de la Maind'œuvre et de la Sécurité du revenu.

Gauthier, M. et L. Mercier (1994). La pauvreté chez les jeunes. Précarité économique et fragilité sociale. Québec: IQRC.

GAUTHIER, M. (dir.) ( 1987). Les nouveaux visages de la pauvreté. Québec: IQRC.

Goedhart, T., V. Halberstadt et B. VAN PRAaG (1977). "The Poverty Line: Concept and Measurement", The Journal of Human Resources, XII: 503-520.

HAGENAARS, A. (1987). "A Class of Poverty Indices", International Economic Review, octobre, vol. 28, $\mathrm{n}^{\circ} 3$ : 583-607.

HAGENAARS, A. et K. DE VOS (1988). "The Definition and Measurement of Poverty", The Journal of Human Resources, vol. XXIII, $\mathrm{n}^{\circ} 2$ : 211-221.

HALBWACHS, M. (1912). La classe ouvrière et les niveaux de vie. Paris: Félix Alcan.

Harp, J. et J.R. Hofley (dir.) (1971). Poverty in Canada. Scarborough: Prentice-Hall.

HANRATTY, M.J. et R.M. BLANK (1992). "Down and Out in North America: Recent Trends in Poverty Rates in the United States and Canada ", The Quarterly Journal of Economics, février: 233-254.

LANGLOIS, S. (1994). "Le niveau de vie des familles. Déclassement et effet de génération ", dans Gilles Pronovost (dir.), Comprendre la famille. Actes du $2^{e}$ symposium québécois de recherche sur la famille. SainteFoy: Presses de l'Université du Québec, p. 17-30.

LANGLOIS, S. (1990). "Pauvreté », dans S. Langlois et coll., La société québécoise en tendances 1960-1990. Québec: IQRC, p. 607-614.

LANGLOIS, S. (1987). "Les seuils de pauvreté », dans Madeleine Gauthier (dir.), Les nouveaux visages de la pauvreté. Québec: IQRC, p. 199-220.

LESEMANN, F. (1994). "La pauvreté : aspects sociaux», dans F. Dumont, S. Langlois et Y. Martin (dir.), Traité des problèmes sociaux. Québec: Institut québécois de recherche sur la culture, p. 581-604.

MARKLAND, S. (1990). "Structures of Modern Poverty", Acta Sociologica, vol. 33, $\mathrm{n}^{\circ} 2: 125-140$.

MAYER, J. et C. JENCKS (1988). "Poverty and the Distribution of Material Hardship", Journal of Human Resources, vol. 24, $\mathrm{n}^{\circ} 1$ : 88-113.

MENDRAS, H. (1990). La seconde Révolution française. Paris: Gallimard.

MINISTÈRE DE LA MAIN-D'CEUVRE ET DE LA SÉCURITÉ DU REVENU (1990). La pauvreté au Québec. Situation et évolution de 1973 à 1986. Québec: Les Publications du Québec.

MORISSETTE, R., J. Myles et G. PICOT (1994). L'inégalité des gains au Canada: le point sur la situation. Ottawa: Statistique Canada, Études analytiques, $n^{\circ} 60$.

NOlan, B. et T. Callan (1994). Poverty and Policy in Ireland. Dublin: Gill \& MacMillan.

PAUGAM, S. (1994). La disqualification sociale. Essai sur la nouvelle pauvreté. Paris: PUF. 
Paugam, S. (dir.) (1996). L'exclusion. L'état des savoirs. Paris: Éditions La Découverte, coll. Textes à l'appui.

PHIPPS, S.A. (1991). "Measuring Poverty Among Canadian Households. Sensitivity to Choice of Measure and Scale», The Journal of Human Resources, vol. XXVIII, $\mathrm{n}^{\circ} 1: 163-184$.

Ravallion, M. (1994). "Poverty Comparison, Gordon Breach», Fundamental of Pure and Applied Economics Series, vol. 56.

RINGEN, S. (1987). "Direct and Indirect Measure of Poverty», Journal of Social Policy, vol. 16, $\mathrm{n}^{\circ} 2: 351-366$.

Ross, D.-P. (1984). Données de base sur la pauvreté au Canada 1983. Ottawa: Conseil canadien de développement social.

SARLO, C. (1992). Poverty in Canada. Vancouver: The Fraser Institute.

SEN, A. (1991). Éthique et économie. Paris: Presses Universitaires de France, coll. Philosophie morale.

SEN, A. (1976). "Poverty: An Ordinal Approach to Measurement ", Econometrica, vol. 44, mars.

SIMMEL, G.(1971). "The poor», dans John Harp et John R. Hofley (dir.), Poverty in Canada. Scarborough: Prentice-Hall, p. 49-74

StANDING, G. (1995). Poverty, Inequality, and the Crisis of Social Policy. Washington: Joint Center for Political and Economic Studies.

StATISTIQue CANAda (1995). Répartition du revenu au Canada selon la taille du revenu 1994. Ottawa: Ministère des Approvisionnements et Services.

TOCQUEVILle, A. de (1835). Mémoire sur le paupérisme (1835), CEuvres, t. 1. Paris: Gallimard, Bibliothèque de la Pléiade, p. 1155-1180.

TOWNSEND, P. (1987). "Deprivation», Journal of Social Policy, vol. 16, $\mathrm{n}^{\circ} 2: 125-146$.

TOWNSEND, P. (1979). Poverty in the United Kingdom. Londres: Penguin.

VAN PRAAG, B. (1977). "The Perception of Welfare Inequality», European Economic Review, 10: 189- 207.

WOLFSON, M.I et J.M. EVANS (1990). Seuils de faible revenu de Statistique Canada. Problèmes et possibilités méthodologiques. Ottawa: Statistique Canada, Document de travail.

WOLFSON, M. (1995). "When Inequality Diverges", American Economic Review, mai.

WOLfSON, M. (1986). "Stasis Amid Change. Income Inequality in Canada 1965-1983», Review of Income and Wealth, décembre, p. 337-369.

ZHENG, B. (1993). "An Axiomatic Characterization of the Watts Poverty Index ", Economics Letters, 42.

ZOUALI, S. (1992). "Incidences de la conjoncture sur la pauvreté et les revenus au Québec et au Canada », Le marché du travail, décembre, p. 73-85. 